Research Article

\title{
Establishment of an Aggregation Model Associated with Instrument Interface Design Based on Kansei Factors of Electric Vehicle Drivers
}

\author{
Yixiang $W u \mathbb{D}^{1}$ and Xinhui Kang $\mathbb{D}^{2}$ \\ ${ }^{1}$ College of Communication and Art Design, University of Shanghai for Science and Technology, No. 516 Jun Gong Road, \\ Shanghai 200093, China \\ ${ }^{2}$ Nanchang University, No. 999 Xuefu Avenue, Nanchang 330031, China
}

Correspondence should be addressed to Yixiang Wu; 3316162376@qq.com

Received 15 July 2019; Accepted 2 December 2019; Published 13 January 2020

Academic Editor: Anna M. Gil-Lafuente

Copyright (C) 2020 Yixiang Wu and Xinhui Kang. This is an open access article distributed under the Creative Commons Attribution License, which permits unrestricted use, distribution, and reproduction in any medium, provided the original work is properly cited.

\begin{abstract}
In traditional Miryoku engineering, the construction of product Kansei factors is only based on the qualitative analysis method. The traditional Miryoku engineering cannot effectively reflect the complex and changeable Kansei factors of users. Therefore, the research path of the Kansei factors needs to be expanded. In this paper, we proposed an evaluation-fuzzy-quantification model based on users' Kansei, and the evaluation analysis, the fuzzy computing, and the quantitative analysis were combined to quantify the importance of design considerations for the instrument interface of electric vehicles. The characteristic of the proposed method is that the qualitative analysis and quantitative analysis are combined to overcome their respective drawbacks. The results of the experiment verified that the proposed method could quantitatively analyze the design consideration factors, and the proposed approach could improve the usability and appeal of the instrument information interface.
\end{abstract}

\section{Introduction}

Since the establishment of Kansei Engineering Society in Japan, Miryoku Engineering has been a part of Kansei Engineering [1]. The Miryoku engineering proposed by Japanese scholars is a user-centered theoretical method to study users' Kansei for product. However, through the study of the relevant literature, there are some shortcomings in the construction users' Kansei factors. It includes the following three points: (1) in the traditional Miryoku engineering, the construction of product Kansei factors is only based on the qualitative analysis method. Therefore, it is necessary to further develop and explore the construction method of the Kansei factors in the traditional Miryoku engineering. (2) The Miryoku engineering has been widely used in the research of product development according to the literature. However, in dealing with the inaccuracy and inexactness of user requirements, the traditional Miryoku engineering cannot effectively reflect the complex and changeable psychological state of users. (3) The framework of traditional Miryoku engineering does not evaluate the importance of Kansei through quantitative analysis, so it is impossible to determine the priority of Kansei in design transformation. Therefore, the research path of the Kansei factors needs to be expanded.

This study takes the instrument interface of electric vehicles as a case. In the space information networks, the sensor is developing towards the integrated information system, of which the interface will not be limited to the existing indicator lights for speed, electric quantity, mileage, and direction, and on which some extra information may be added. To rapidly and precisely obtain comprehensive information through the instrument interface, the visual appeal-oriented interface design is very important, especially for the driver and the monitoring personnel on the ground.

Due to the lightweight requirement for sensors, the sensor information interface must achieve the visual performances of both visibility and readability, and the sensor 
information interface's space would concurrently be reduced. Whether the person can intuitively judge the information displayed on the screen while driving is related to the comprised elements on the sensor interface, the special arrangement of the elements, the size, and the proportion. With respect to the design of the sensor information interface, good visibility, wonderful visual appeal, small size, and lightweight requirements should all be taken into consideration. The display modes on the sensor information interface could be flexible. Many information elements on the sensor information interface may be represented with new forms and increasingly more information can be added to the sensor information interface. An important problem that should be studied is that the sensor information interface design should accord with the personal customs and characteristics of the sensor. With some new sensors, the imitated traditional design should not be continued. Additionally, even with the existing sensor, the sensor information interface design should also be adjusted to meet users' preferences.

In this paper, the author proposed a model based on users' Kansei to determine the priority of Kansei factors of the instrument interface of electric vehicles. In this model, the evaluation analysis, the fuzzy computing, and the quantitative analysis were combined to quantify the importance of design considerations for the interface. The results of the experiment verified that the proposed method could quantitatively analyze the design consideration factors and the proposed approach could improve the usability and appeal of the instrument information interface.

\section{Related Work}

With the modern sensor information interface becoming more complicated due to their new functions and elements, [2] the service and information of the sensor interface (including safety systems, personal communications, entertainment, and location information) should not distract the personnel from their attention on the main objects. Then, the design of sensor interfaces should provide the correct information for the personnel, be clearly readable, and be rapidly understood [3]. Therefore, the design of the sensorial interface is requested to meet the demand that users could rapidly get the ideas. Owsley et al. [4] proposed that reducing the contrast ratio and spatial resolution might result in a visibility-related problem when personnel were reviewing the icons on the sensor interface. Gibson et al. [5] argued that the effectiveness, visibility, and comfortable operations are really important for the sensor interface.

Gkouskos [6] utilized conceptual vehicles to discover the constructs of user needs. Through their study, nineteen necessary dimensions were revealed: automation, calmness, comfort and convenience, connectivity, control, driver support, trip context, driving pleasure, efficiency, environmental impact, freedom of choice, interaction fluency, ownership, personalization, safety, self-image, simplicity, technology, and versatility. Xue et al. [7] provided the instructions for the color saturation design of the interface. The research results of Ells and Dewar [8] suggested that people's responses to the symbolic pattern are quicker than that to the character and number and better responses may appear in bad audio-video circumstances. Since different design objectives should adopt different manners of presenting the information, number and character may be better for some objectives while patterns may be better for others. The ultimate goal is allowing the users to read the information in the fastest and clearest way.

A sensor interface with better usability and appeal must also boost the competitiveness of the sensor. [9] Therefore, the design of the sensor interface is receiving increasingly more research attention. Liu et al. [10] proposed that the sensor interface should be equipped with better methods of design and configuration so that personnel may easily receive information and a great quantity of differential information on the interface may be greatly reduced, thus accomplishing the purpose of increasing the safety and stability of driving. Gibson et al. [5] proposed that the key issue is that companies should conduct user-centered designing and the analysis by which interface shall meet the requirements of markets and users.

To meet the customized requirements of the interface, several common techniques (including the Kansei engineering, [11] the evaluation grid method (EGM) [12], and the fuzzy analytic hierarchy process (FAHP) [13]) have been proposed to reduce the gaps between customer requirements and sensor interfaces.

Ahmed and Yannou [14] proposed that the design attributes of sensors and the users' perceptions are intertwined and correlated with each other. They also proposed that Japanese researchers used the scientific research methods of Kansei Engineering to study the correlation between the physical attributes and perceptual attributes of a sensor interface. For example, Jindo and Hirasago [15] divided the components of the interface into the following factors: the type of sensor, the character pattern, the shape of the pointer, and the type of the dimension. Smith and Fu [16] explored the relationships between the presentation image designs of a sensor interface and personnel's Kansei responses. They built a prediction model that described the relationships between the representative Kansei factors and physical image design properties of the sensor interface for the future user-centered presentation image design of sensor interfaces. Ho and Hou [17] stated the design strategy for the attractiveness of the interface, and $\mathrm{Ma}$ et al. [18] presented the evolution of the preference-based design.

The greatest advantage of the EGM is that the structure of users' Kansei factors can be elicited [19, 20]. The repertory grid method developed by Kelly can be used to capture the mechanism of people's comprehension and recognition of their environments, especially in human relationships [21]. Sanui [22] advanced it into the evaluation of the grid method in two processes. This method mainly discusses the similarities and differences among the objects to sort out the target object of individual qualities through personal interviews and the paired comparison between objects A and B [22]. The qualitative EGM can be used to build the abstract feelings of interviewees specific to a kind of real feeling [12]. The results are expressed by using the hierarchical diagram 
of the appeal factors for evaluation [23]. By researching the attractive factors of app icons, Ho and Hou found that the EGM is a quantitative method for analyzing the influences of design factors [17]. Park et al. [24] disclosed the relationship between the specific attribute and ultimate value when using a mobile hospital application via the implementation of the EGM based on further interviews to various ages of people.

Saaty [25] developed the analytic hierarchy process (AHP) to help decision makers make consistent judgments. AHP is a kind of mathematical tool capable of reducing a priority vector from a consistent matrix [26]. The priority vector is called the leading feature vector to show the sequence of the consumers' preferences during product development decision [27]. Meanwhile, the AHP is a tool widely applied in product design development, usability evaluation, and other research fields. Chakraborty et al. [26] evaluated the remanufacturing strategies via the Analytic Hierarchy Process (AHP) to calculate the weight of the corresponding design criteria, thus bringing the best design method for the remanufacturing. Sapuan et al. [27] have used the AHP for determining the most suitable material for the automotive dashboard panel. Combined with the AHP and fuzzy evaluating method, Zhou and Chan [28] proposed an integrated evaluation method for products availability. The AHP offers greater benefits and helps the design engineer to identify the most and least important requirements for the product design [29].

Due to its uncertainty and vagueness in the judgment of those customer requirements, AHP seems to be specified insufficiently and inaccurately to reduce the importance rates of consumers' requirements [29]. Specific to the inaccuracy of the AHP, Van Laarhoven and Pedrycz [30] proposed a fuzzy analytic hierarchy process (FAHP). The FAHP has been used by many researchers, such as, Chen [31] built a decision-making support model for the visual design of perfume bottles via the FAHP, so as to provide a new method for perfume package designers. To choose the best digital camera for consumers, Alptekin built a decision support framework according to the subjective evaluation of the consumers and photography experts based on the fuzzy set theory and fuzzy AHP [32]. By using the FAHP, Tu and $\mathrm{Hu}$ [33] constructed the Product Innovative Service System of Wedding Photography Apparel. Based on consumers' emotional response, the above two traditional decision models are usually used to integrate the opinions of the group via the arithmetic mean or geometric mean, but its results are unreasonable to some extent. The formation of experts consensus is the key point of group decision-making. Therefore, to solve such problem, Hsu and Chen [34] proposed the Similarity Aggregation Method (SAM) to develop a set of fuzzy multicriteria decision model combined with the fuzzy AHP. The similarity measurement of the fuzzy number is a very important topic in group decision-making, when group decision makers have the same mode of thinking, but fuzzy opinion, the similarity of the expert opinion can be expressed by the fuzzy theory concept. A few of the research articles integrated the SAM with FAHP and EGM for analyzing the availability and visual attraction factors of the instrument interface. In this study, the EGM is applied to build the hierarchical diagram, and moreover, the SAM is utilized to integrate the opinion of experts. Afterwards, the FAHP is applied to calculate the weight of each factor, and finally, according to the defuzzification results, the priority of Kansei factors of the instrument interface is ranked.

\section{The Optimization Method of the Instrument Interface Design Based on Users' Kansei}

In this paper, we adopt the method of the instrument interface design based on the users' Kansei preference. To analyze the factors of usability and visual attractiveness for the instrument interface, the author proposes an aggregation method to make the analysis, as shown in Figure 1. First, the evaluation phase is utilized to build the hierarchical diagram. The upper level of this diagram is the psychological feelings of users, the lower level is the specific characteristics of the sensor interface, and the middle level is the specific reasons for the attraction. Then, at the user preference calculation phase, the upper-level factor gained at Stage I is taken as the evaluation criterion for importance analysis. The opinions of all experts are first integrated through SAM, and finally a fuzzy paired comparison matrix is built. According to this matrix, the weight of evaluation criteria is calculated with the Fuzzy AHP method; thus, the upper-level key Kansei factors are determined based on the accurate calculation. Finally, the hierarchical structure is built based on key factors (Kansei image), to analyze the weight relationship between the upper level, middle level, and lower level. The proposed aggregation model is shown in detail as follows.

\subsection{Stage 1: Construction of the EGM Hierarchical Diagram} through Qualitative Analysis. Ten users with more than 5 years' experience in electric vehicle driving are invited for testing. The test time of each testee lasts 20 minutes. The test venue is in a separate room without external interruption. To realize more real effect, notebook computer display instrument is used as the sample and matches with the projector for driving environment projection, in addition to an electric vehicle steering wheel to establish a basic simulation experimental environment. The film projected by the projector is the roadside scenario during driving. The notebook screen inclination display is in accordance with the angle of the instrument board observed by the real driver. The seat height of the testee is the same as the actual seat height when driving for testee immersion to the entire experimental scenario.

The special procedures for applying evaluation grid method (EGM) to discover users' Kansei image preferences for information interfaces are listed as follows.

(1) The simulation test is performed according to the testee's habit of scanning information in the instrument interface when driving the electric vehicle. After the test, the testee is asked to sort out one to three samples that they consider attractive based on the simulation feeling just now. 


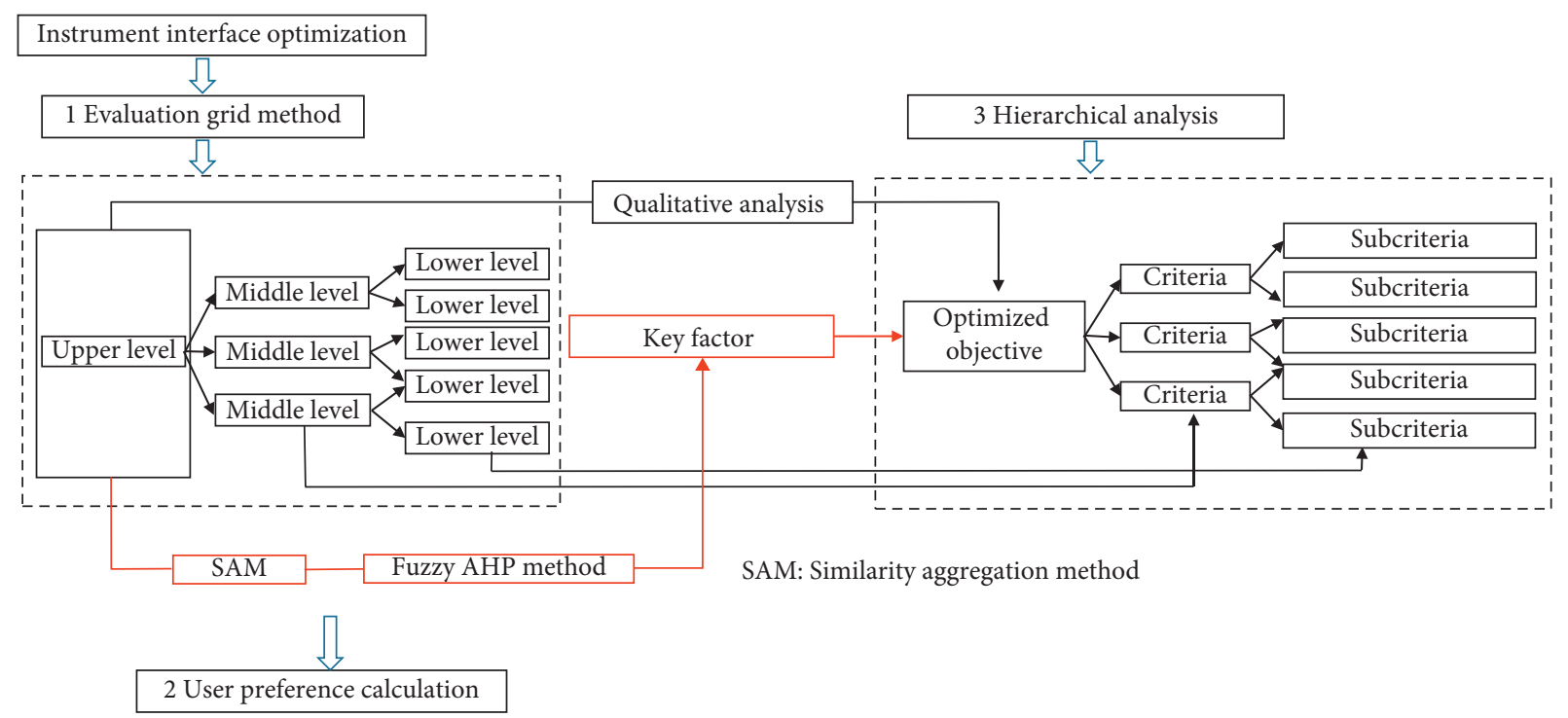

FIGURE 1: The proposed aggregation model.

(2) According to the one to three attractive samples selected by the testee, the testee is inquired to answer why these samples are attractive, and the reasons will be the original evaluation items. For example, the information display mode of the instrument speedometer which is deemed attractive by the testee will be denoted as original evaluation items (middle level).

(3) The "upper level" and "lower level" for each of the original evaluation items will be obtained by continuous inquiry. The "upper level" in the EGM hierarchical diagram represents the psychological feeling of the testee, and the "lower level" represents the specific characteristic that the instrument sample of the electric vehicle attracts the testee. For example, if the information display mode of the speedometer is considered attractive due to the specific feature of displaying the lower level by a number, which makes the testee feel easy to recognize psychologically, those discernible can be the "upper level," as shown in Figure 2.

(4) The hierarchical diagram of each testee can be constructed by repeating the above process. After merging approximate descriptions by comparing the hierarchical diagram of all testees, the EGM hierarchical diagram of the EV instrument interface that fits all the testees' Kansei image preference can be sorted out.

3.2. Stage II: Determining Key Upper-Level Factors through Quantitative Analysis. While the Kansei factors attained through the evaluation phase constitute the basic evaluation, the in-between importance ordering and the quantitative weight factors are unclear. Thus, the fuzzy-paired comparison matrix is established with the SAM in this stage for the "upper-level" factors (Kansei images) attained by the evaluation phase, and then weight calculation and ranking are carried out for Kansei images, so as to obtain Kansei images ranking first.

3.2.1. Expert Questionnaire. Experts are first invited for paired comparison of relative importance between any two Kansei images, to gain the value of the relative importance between any two Kansei images, and this may help build the paired comparison matrix. The nine-point evaluation scale of Saaty is adopted in the questionnaire, as shown in Figure 3.

Then, the triangular fuzzy value is established. In the paired comparison matrix, each value denotes the expert opinions on each testee. The triangular fuzzy number is based on this study to transform the value of the relative importance of the paired comparison between evaluation items from experts into the fuzzy number, as shown in Table 1 below.

3.2.2. Applying the SAM to Integrate Expert Opinions. In order to integrate the fuzzy evaluation value of the expert and obtain the importance comparison fuzzy values of any two criteria $a$ and $b$, the Consensus Degree Coefficient (CDC) of each expert is calculated by applying the SAM [34]. The following steps show this procedure in detail $[34,35]$ :

Step 1: the SAM aggregates individual fuzzy opinions into a group fuzzy consensus opinion. The agreement degree $\left(S\left(\widetilde{R}_{p}, \widetilde{R}_{q}\right)\right)$ of any two experts is calculated as follows. $\widetilde{R}_{p} \stackrel{=}{=}\left(L_{p}, M_{p}, U_{p}\right)$ and $\widetilde{R}_{q}=\left(L_{q}, M_{q}, U_{q}\right)$ are set to represent the opinion of expert $E_{p}$ and $E_{q}$, $p=1,2, \ldots, n$. The following area ratio defines the agreement degrees of experts $E_{p}$ and $E_{q}$ :

$$
S\left(\widetilde{R}_{p}, \widetilde{R}_{q}\right)=\frac{\operatorname{area}\left(\widetilde{R}_{n_{p}} \cap \widetilde{R}_{m_{q}}\right)}{\operatorname{area}\left(\widetilde{R}_{n_{p}} \cup \widetilde{R}_{m_{q}}\right)} .
$$




Middle level

Figure 2: The EGM hierarchical diagram with an item.

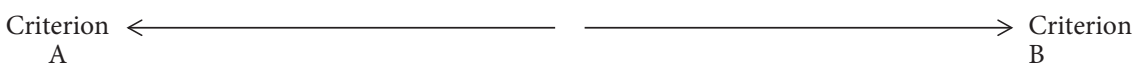

FIGURE 3: Evaluation on relative importance between Kansei images for paired comparison.

Table 1: Comparison between the triangular fuzzy number and its fuzzy linguistic scale end point.

\begin{tabular}{lcc}
\hline Fuzzy number & End point of fuzzy number & Linguistic scale \\
\hline$\widetilde{1}$ & $(1 / 2,1,2)$ & Equally important \\
$\widetilde{2}$ & $(1,2,3)$ & Between equally important and slightly important \\
$\widetilde{3}$ & $(2,3,4)$ & Slightly important \\
$\widetilde{4}$ & $(3,4,5)$ & Between slightly important and important \\
$\widetilde{5}$ & $(4,5,6)$ & Important \\
$\widetilde{6}$ & $(5,6,7)$ & Between important and very important \\
$\widetilde{7}$ & $(6,7,8)$ & Very important \\
$\widetilde{8}$ & $(7,8,9)$ & Between very important and extremely important \\
$\widetilde{9}$ & $(8,9,9)$ & Extremely important \\
\hline
\end{tabular}

Step 2: an agreement matrix (AM) is constructed based on the agreement degree and is expressed as equation (2), where if $p=q, S_{p q}=1$ and if $p \neq q, S_{p q}=S\left(\widetilde{R}_{p}, \widetilde{R}_{q}\right)$ :

$$
\mathrm{AM}=S_{p q} \times n, \quad p q=1,2, \ldots, n .
$$

Step 3: the average agreement degree $\left(A\left(E_{p}\right)\right)$ of expert $E_{p}, p=1,2, \ldots, n$, is calculated by the AM, as shown in the following:

$$
A\left(E_{p}\right)=\frac{1}{n-1} \sum_{p=1, p \neq q}^{n} S_{p q}, \quad p, q=1,2, \ldots, n .
$$

Step 4: then, the relative agreement degree $\left(\mathrm{RAD}_{p}\right)$ of expert $E_{p}, p=1,2, \ldots, n$, is computed as below:

$$
\operatorname{RAD}_{p}=\frac{A\left(E_{p}\right)}{\sum_{p=1}^{n} A\left(E_{p}\right)}, \quad p, q=1,2, \ldots, n .
$$

Step 5: the consensus degree coefficient $\left(\mathrm{CDC}_{p}\right)$ of expert $E_{p}, p=1,2, \ldots, n$, is calculated, as shown in equation (5), with $0 \leq \beta \leq 1$ :

$\mathrm{CDC}_{p}=\beta \times w_{p}+(1-\beta) \times \mathrm{RAD}_{p}, \quad p, q=1,2, \ldots, n$.

However, the degree of importance of each expert $(\beta=0)$ is not taken into account in the decision analysis process of this study, and $\mathrm{CDC}_{p}=\mathrm{RAD}_{p}$ represents the consensus degree coefficients of the experts $E_{p}, p=1,2, \ldots, n$.

Step 6: the fuzzy evaluation value of all experts is integrated, as shown in (6). $\widetilde{R}_{a b}$ represents the fuzzy number by integrating experts' opinion for any two criteria or subcriteria $a$ and $b$ and $\widetilde{R}_{a b}^{p}$ is the fuzzy evaluation value of the $p$ th expert after appraising the two criteria or subcriteria $a$ and $b$ :

$$
\widetilde{R}_{a b}=\sum_{p=1}^{n} \mathrm{CDC}_{p} \otimes \widetilde{R}_{a b}^{p}, \quad p, q=1,2, \ldots, n .
$$

\subsubsection{Using the FAHP to Calculate the Weight of Each Kansei Image}

Step 1: fuzzy analytic hierarchy process (FAHP) was proposed by Professor T. L. Saaty, an American operations' research expert. In order to calculate the weight value of a pair of evaluation items, the fuzzy pairwise comparison matrix $\widetilde{A}$ is established based on the fuzzy number $a_{i j}$. Each fuzzy number $a_{i j}$ represents that the data is closer to $a_{i j}$, while each membership function is defined by three symmetric triangular fuzzy numbers, which are, respectively, represented by the left, middle, and right numbers in the range of definition. $a_{i j}=0.5$ represents that the factor is as important as itself; $a_{i j} \in[0.1,0.5)$ represents that the factor $x_{j}$ is more important than $x_{i} ; a_{i j} \in[0.5,0.9)$, which means that the factor $x_{i}$ is more important than $x_{j}$. Based on the numerical scale above, the following fuzzy pairwise comparison matrix is obtained according to the mutual comparison of factors $a_{1}, a_{2}, \ldots, a_{n}$ :

$$
A=\left[\begin{array}{cccc}
a_{11} & a_{12} & \cdots & a_{1 n} \\
a_{21} & a_{22} & \cdots & a_{2 n} \\
\cdots & \cdots & \cdots & \cdots \\
a_{n^{1}} & a_{n^{2}} & \cdots & a_{m}
\end{array}\right] .
$$


In this study, the fuzzy pairwise comparison matrix $\widetilde{A}$ is established by using the similarity aggregation method (SAM) and FAHP; therefore, the fuzzy number in $\widetilde{A}$ is derived from formulas (1)-(6) in the calculation steps of the SAM. In the SAM, the degree of agreement between each decision maker is employed to integrate the evaluation values of multiple decision makers through similarity function. After integrating the evaluation values of several decision makers for a pair of evaluation items, a fuzzy number in the matrix $\widetilde{A}$ is obtained. By repeating the above steps with the SAM, the matrix $\widetilde{A}$ of the FAHP can be constructed, then the weight of each evaluation item is calculated by the FAHP, and finally the evaluation criteria are sorted.

Step 2 (weight formula of fuzzy pairwise comparison matrix): after the fuzzy pairwise comparison matrix is established, in order to obtain the weight of each attribute in the final evaluation result, the geometric mean, weight, $\lambda_{\text {max }}$, etc., can be calculated as the weight of the fuzzy pairwise comparison matrix. In this study, the column vector geometric average method is used to obtain the weight of the fuzzy pairwise comparison matrix. First, the geometric mean value of each row is calculated according to the fuzzy pairwise comparison matrix, as shown in the following formula:

$$
Z_{i}=\left(a_{i 1} \otimes a_{i 2} \otimes \cdots \otimes a_{i n}\right)^{1 / n},
$$

where $Z_{i}$ is the geometric mean value of each row of the fuzzy pairwise comparison matrix and $a_{i j}$ stands for each element of the fuzzy pairwise comparison matrix.

After obtaining the geometric mean $Z_{i}$ of each row of the fuzzy pairwise comparison matrix, the weight of each row of the fuzzy pairwise comparison matrix is solved, as shown in the following formula:

$$
W_{i}=Z_{i} \otimes\left(Z_{1} \oplus Z_{2} \oplus \cdots \oplus Z_{n}\right) .
$$

According to the above method, the fuzzy weight vector $W$ of the fuzzy pairwise comparison matrix can be obtained, as shown in the following formula:

$$
W=\left(W_{L}, W_{M}, W_{U}\right), \quad i=1,2, \ldots, n
$$

where the fuzzy number $W$ of each weight is a triangular fuzzy number and $W_{L}, W_{M}$, and $W_{U}$ are, respectively, represented by the left, middle, and right numbers in the range of definition $W$.

Step 3 (consistency check of fuzzy pairwise comparison matrix): the consistency check of the fuzzy pairwise comparison matrix should be carried out to check whether the weight value obtained in Step 2 is reasonable. When the deviation consistency is too large, it indicates that it is unreliable to use the calculation result of the weight vector as the decision basis. Prior to the consistency check, the maximum eigenvalue of the fuzzy pairwise comparison matrix should be calculated, as shown in the following formula:

$$
A W=\lambda_{\max } W
$$

where $A$ is a fuzzy pairwise comparison matrix established by FAHP, $W$ the fuzzy weight vector of the fuzzy pairwise comparison matrix, and $\lambda_{\max }$ the maximum eigenvalue of the fuzzy pairwise comparison matrix.

The rationality of the comparison matrix of the respondents' response results is verified by defining the consistency index CI and CR. Generally, CI $\leq 0.1$ is an acceptable error and the maximum allowable range is $\mathrm{CI} \leq 0.2$. The formula for $\mathrm{CI}$ is as follows:

$$
\mathrm{CI}=\frac{\lambda_{\max }-n}{n-1}
$$

$\lambda_{\max }$ stands for the maximum eigenvalue of the comparison matrix. The closer it is to $n$ (the number of elements in the matrix), the more consistent it is. The consistency ratio (CR) is verified as the ratio of the values of $\mathrm{CI}$ and $\mathrm{RI}$ in the same matrix order, as shown in the following formula:

$$
\mathrm{CR}=\frac{\mathrm{CI}}{\mathrm{RI}}
$$

If C.R. is less than or equal to 0.1 , the fuzzy pairwise comparison matrix is consistent; according to the number of elements (order $n$ ), each comparison matrix corresponds to a random index (random index, R.I.), as shown in Table 2.

After the consistency check of the fuzzy pairwise comparison matrix, it is reliable to take the calculation result of the weight vector as the decision basis.

Step 4 (comprehensive evaluation index): In order to facilitate the comparison of the final evaluation values for the importance of each adjective, the fuzzy weights of each initial criterion are defuzzified by a simple centroid method to obtain a single defuzzification value as the comprehensive evaluation index. The weight $W_{L i}$ is subtracted from the weights $W_{M i}$ and $W_{U i}$, respectively, the two calculated results are added and then divided by 3 , and finally, we obtain the final defuzzification result by adding the weight $W_{L i}$. The specific calculation method is shown below:

$$
D F_{i}=\frac{\left[\left(W_{U i}-W_{L i}\right)+\left(W_{M i}-W_{L i}\right)\right]}{3}+W_{L i},
$$

where $F_{i}$ is the $i$ th fuzzy weight vector. The specific defuzzification value $D$ of each perceptual factor is obtained through the above defuzzification method and is used as a comprehensive evaluation index for evaluating the importance of each perceptual factor. After the application of the FAHP, the qualitative and quantitative methods are combined to obtain reliable indexes that can reflect the importance of each 
TABLE 2: Random index [35].

\begin{tabular}{|c|c|c|c|c|c|c|c|c|c|c|c|c|c|c|c|}
\hline$n$ & 1 & 2 & 3 & 4 & 5 & 6 & 7 & 8 & 9 & 10 & 11 & 12 & 13 & 14 & 15 \\
\hline R.I. & 0 & 0 & 0.58 & 0.90 & 1.12 & 1.24 & 1.32 & 1.41 & 1.45 & 1.49 & 1.51 & 1.48 & 1.56 & 1.57 & 1.59 \\
\hline
\end{tabular}

perceptual factor. This method is effective in solving complex and uncertain problems.

$\mathrm{Wu}$ and Cheng [35] adopted the FAHP to prioritize the eight design styles of the product form (the target level of the hierarchical structure), such as fashionable and technological style, according to the weight value. However, only the FAHP is used to calculate the fuzzy weight values of the eight design styles (the upper level of the hierarchical structure), without building the hierarchical structure model for the target (the design style of the product form). While the core idea of the FAHP is to build a hierarchical structure model for the problem to be solved. Therefore, we first used the evaluation grid method (EGM) to obtain the upper-level emotional factor, the middle-level emotional factor, and the lower-level emotional factor of the electric vehicle driver, and then used the FAHP method to establish the three-level structure of the emotional factor in this study. This is the first time that the AHP is used to conduct quantitative analysis on the basis of qualitative analysis in the same kind of research (EGM). Compared with the previous research studies, this research is more innovative as follows. First, the three-level architectural relationship can be established by the EGM, which cannot quantitatively analyze the relationship between the three levels, but can be used as the evaluation index of three levels of the AHP. Therefore, it is novel to use the three-level architecture by the EGM to establish the AHP. Second, the FAHP is used to not only prioritize the upper level (target level) of the hierarchical structure according to the weight value but also calculate the weight relationship between the middle and lower levels, and finally through the series connection of the weight valve, the specific characteristics of the instrument interface that affect the electric vehicle driver's emotional factor are found in this study. In terms of method, this study differs from the study of $\mathrm{Wu}$ and Cheng [35] in which a hierarchical structure of the problem to be solved is systematically built and a threelevel structural model is constructed according to the interrelated influence and affiliation between the levels. In this way, the problem is concluded to the determination of the relatively important weight value of the lower level (specific characteristics of the instrument interface, etc.) relative to the upper level (the driver's emotional factor) or the arrangement of the relative priority.

3.3. Stage III: Establishing the Hierarchical Structure of Key Upper-Level Factors. According to the weight ranking result of Stage II, the upper-level factor (Kansei image) ranking first can be obtained. Based on the hierarchical diagram constructed by EGM at Stage I, a hierarchical structure can be constructed between the corresponding middle-level factors of the upper-level factors ranking first and the lowerlevel factors, as shown in Figure 4.
The fuzzy analytic hierarchy process (FAHP) expert questionnaire is made. In this questionnaire, the key upperlevel factor ranking first is deemed as the objective of the optimized design of the instrument interface, each middlelevel factor as the evaluation criterion and each lower-level factor as the subcriterion. 12 experts are invited to compare any two between evaluation criteria and between subcriteria included. For example, in order to make the instrument interface of the electric vehicle to satisfy the optimized objective of "making the information distinguishable," suppose the evaluation criteria include $\mathrm{C} 1, \mathrm{C} 2, \mathrm{C} 3, \mathrm{C} 4$, and $\mathrm{C} 5$, and $\mathrm{C} 1$ relates to the evaluation subcriteria, which involve $\mathrm{C} 11, \mathrm{C} 12, \mathrm{C} 13$, and C14. As shown in Figures 5 and 6, 12 experts are invited to compare any two between evaluation criteria $(\mathrm{C} 1, \mathrm{C} 2, \mathrm{C} 3, \mathrm{C} 4$, and $\mathrm{C} 5)$ and any two between evaluation subcriteria (C11, C12, C13, and C14) under C1.

For the comparison between any pair of evaluation criteria or subcriteria for the importance, equations (1)-(6) are used to integrate the fuzzy numbers of all experts to form an expert consensus. The integrated fuzzy number is input into the fuzzy paired comparison matrix, and then equations (7)-(14) are used to calculate the weights of each evaluation criteria and its subcriteria. The weight of the criterion is multiplied by the weight of the subcriteria it includes, and the hierarchical concatenation is performed to obtain the weights of all the lower-level factors and sort them. The author calculates the weights of the factors that have attraction against the electric vehicle instrument interface by integrating the similarity aggregation method (SAM) and the fuzzy analysis hierarchy process (FAHP), and then the importance of each factor is derived, thereby determining the weighting relationship between the lower-level factors (specific characteristics) and the upper-level factor (optimization object).

\section{Results and Analysis}

4.1. Stage I: The Evaluation Phase. The users with more than 5 years' experience in electric vehicle driving are evaluated by the EGM in this study to determine the factors that testees consider the electric vehicle instrument interface attractive and to construct a hierarchy diagram of these attractive factors (namely, the hierarchical diagram). See Section 3.1 for the special procedures for applying the EGM to discover users' preferences for information interfaces. Based on the EGM processes, the evaluation grid diagram of each testee can be constructed. The hierarchical diagram of a testee's Kansei image preference for the electric vehicle instrument interface is shown in Figure 7.

By analyzing and comparing the grid diagram of 10 testees' evaluation, it is available to simplify words with similar meanings via group discussion and merge these Kansei images based on the similarity, as shown in Figure 8. 


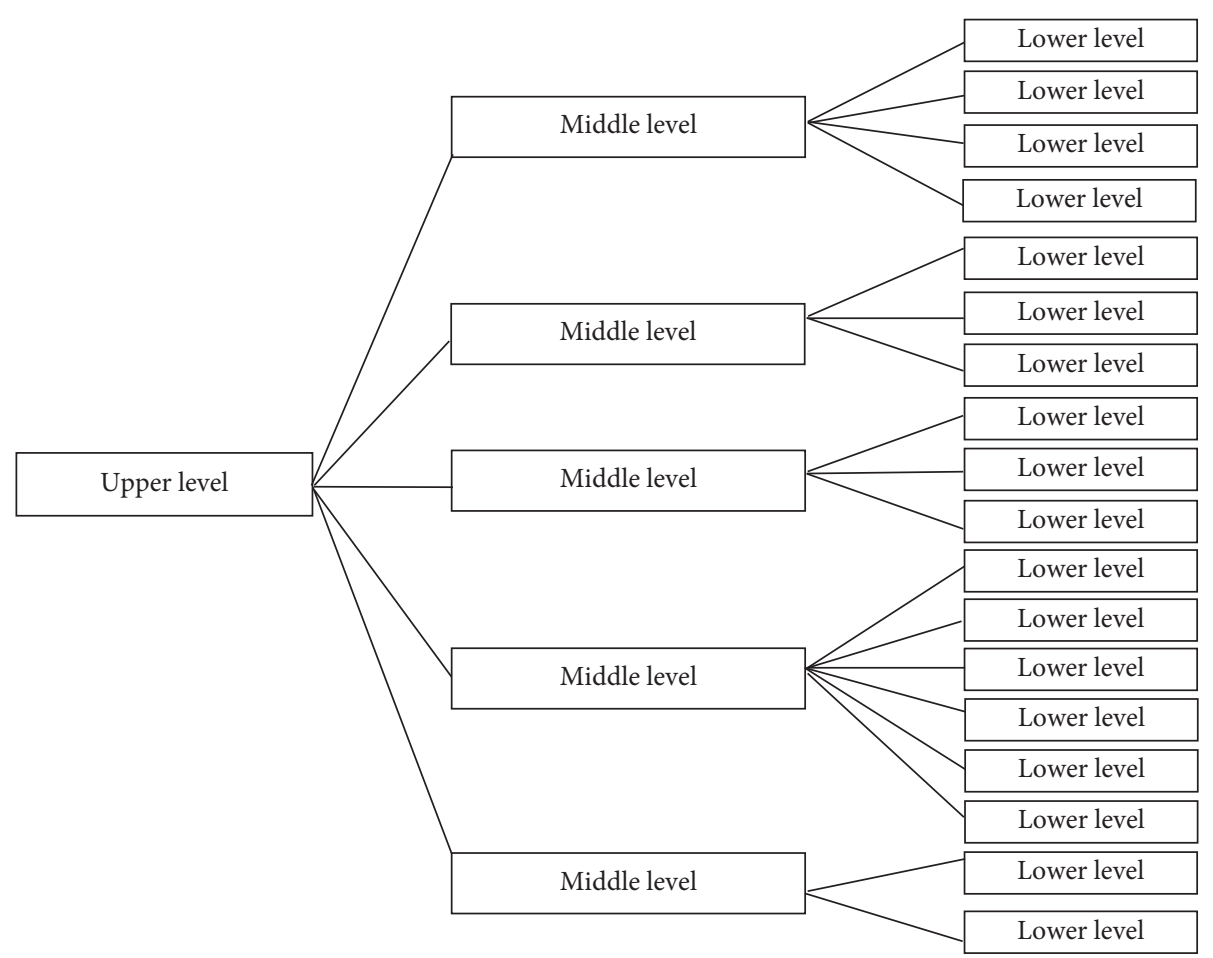

FIgURE 4: The corresponding hierarchical diagram of the upper-level factor (Kansei image).

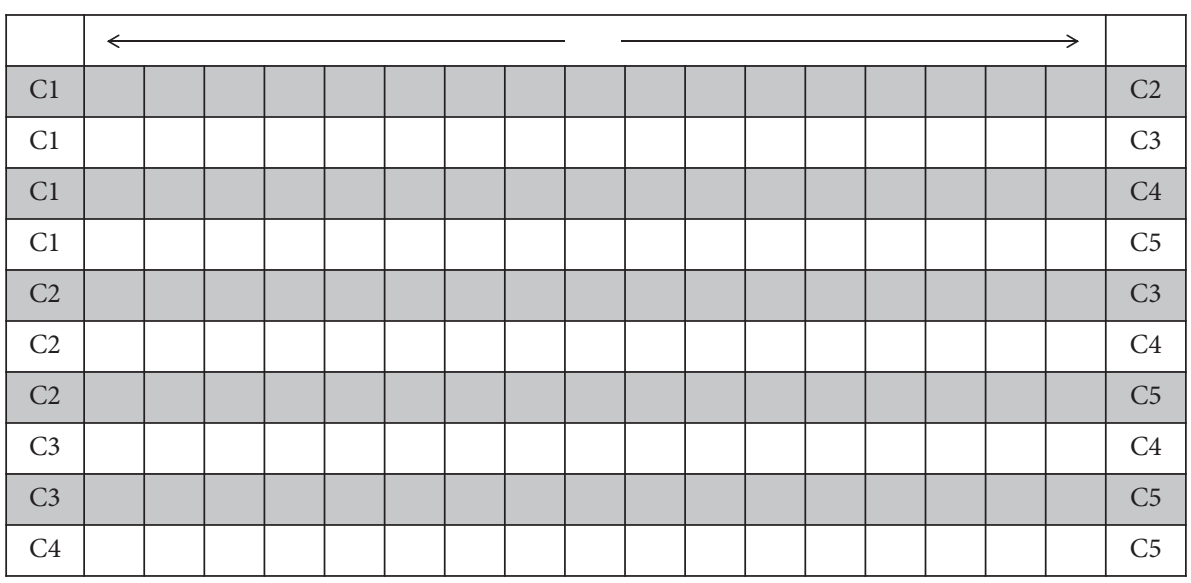

Figure 5: Pairwise comparison between the evaluation criteria.

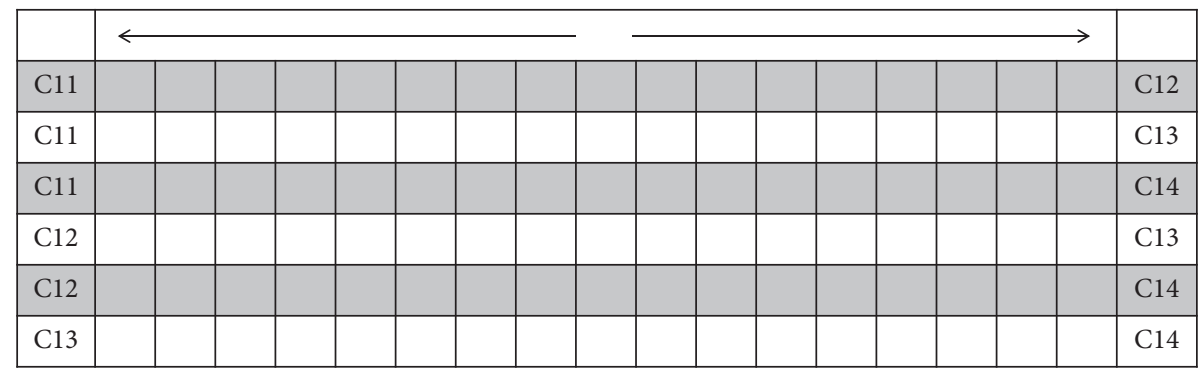

Figure 6: Pairwise comparison between the evaluation subcriteria.

Ten upper-level factors are obtained in this study. The top six factors among ten upper-level factors are Easy to Recognize, Concise and displaying-Visual, Distinct difference between the primary and secondary part, Striking, Practical, and Futuristic. Similar descriptions of the middle level and the lower level of ten testees can be 


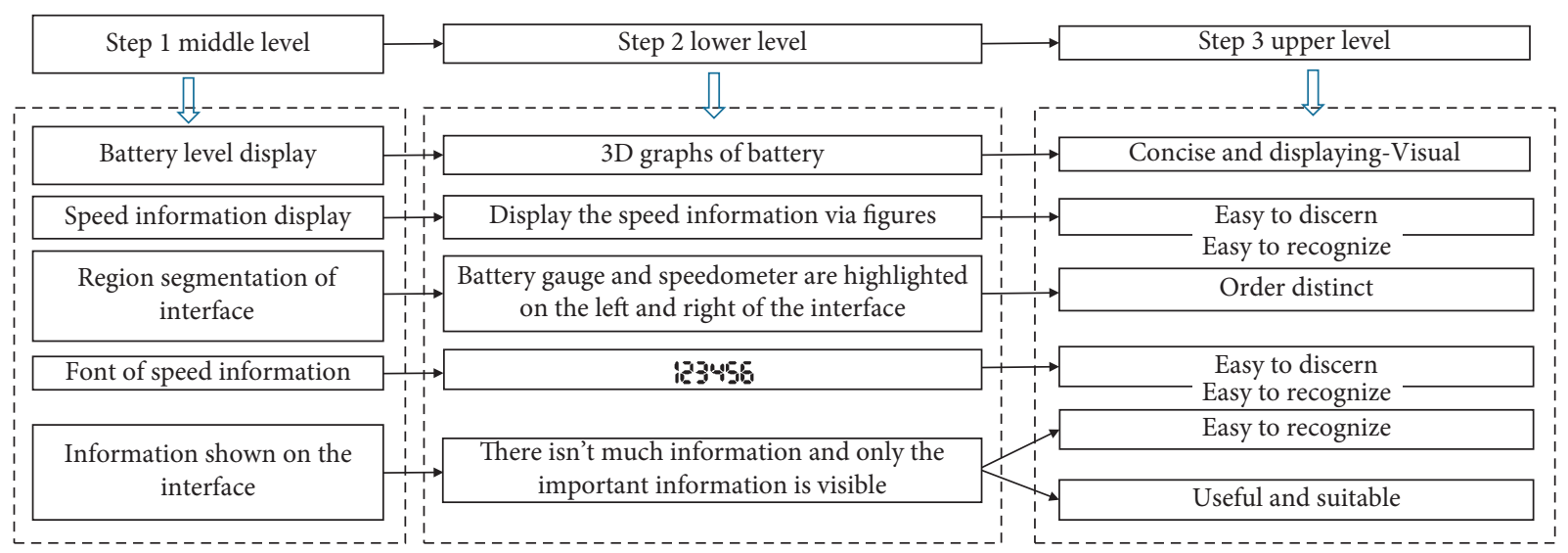

Figure 7: Grid diagram of testee 1's evaluation.

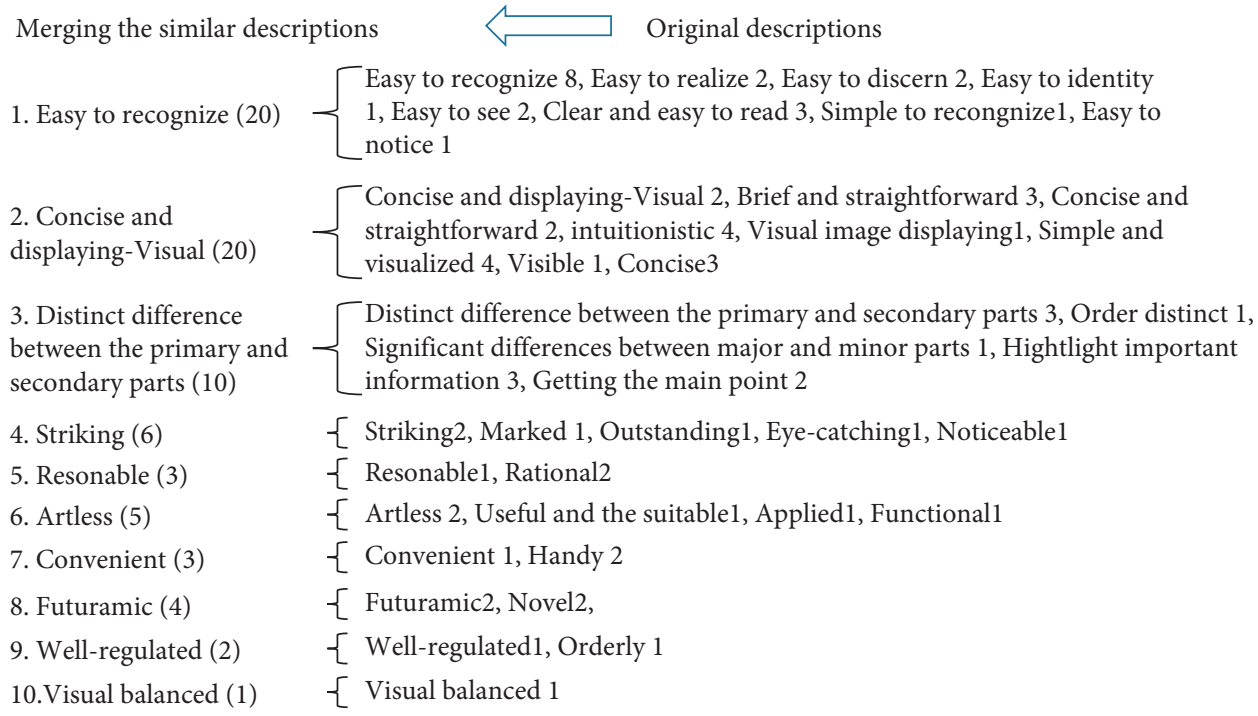

FIGURE 8: Integration of the descriptions of similar Kansei images.

simplified by using the same simplification method. The evaluation grid diagram of all the testees is systemized, as shown in Figure 9. To take factors easy to recognize in the upper level as the example, corresponding factors in the middle level can be found according to the connecting lines in the figure, including C1 (Speed information display), C2 (Font of speed information), C3 (Battery level display), C4 (Region segmentation of interface), and C5 (Interface information); factors in the lower level include C11 (Visually display the speed information via the shaded scale bars), C12 (Display the speed information via figures), C13 (Simultaneously display the speed information via the scale + number), C14 (Warn the high-speed region via color blocks), C21 (Font of electronic meter), C22 (Italics), C23 (Font size), C31 (Battery level + percentage display), C32 (3D graphs of battery), C33 (indication of the battery level via 10 bars), C41 (battery gauge and speedometer are highlighted on the left and right of the interface), C42 (speedometer is configured centrally and surrounded by other parameters), C43 (three regions of the battery gauge, speedometer, and other functional zone are evenly distributed on the interface comparatively), C51 (the instrument interface displays the primary information, such as battery level, speed per hour, milometer, and signal light), C52 (besides indicating the primary information, the instrument interface further displays the secondary information, such as GPS, Bluetooth, photosensitive-induction headlamp, and network signal), and C53 (the instrument interface displays lots of comprehensive information).

4.2. Determining Key Kansei Images. In this study, the author uses the EGM to collect Kansei images of the electric vehicle's instrument interface for further quantitative research. The upper-level factors ranking top 6 obtained by the EGM are only the result of qualitative analysis, while the weight of each factor cannot be gained. Therefore, the upperlevel factors obtained by the EGM are used as 6 evaluation criteria in this study. The opinions of all specialists are first integrated through the SAM, and finally a fuzzy-paired comparison matrix is built. According to this matrix, the 


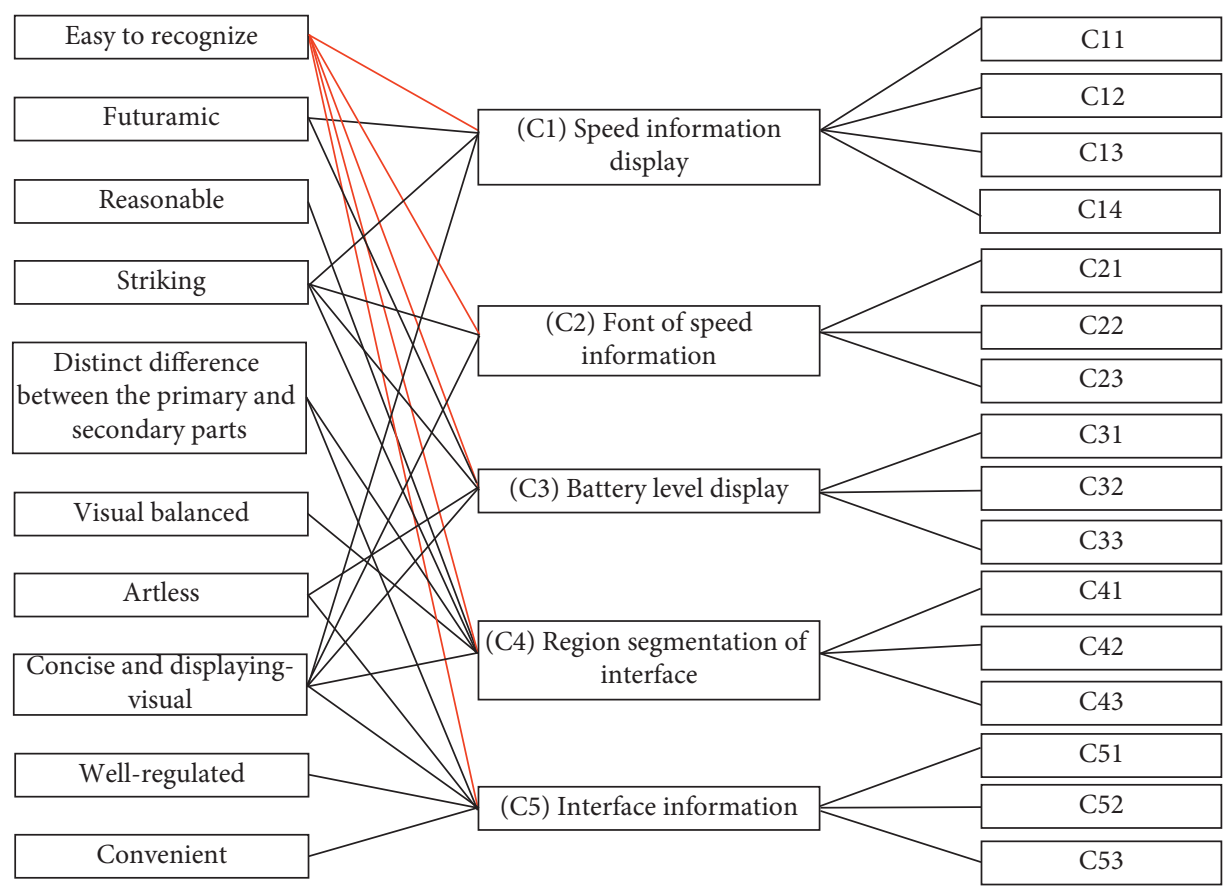

FIGURE 9: The hierarchical diagram of Kansei image preferences for the instrument interface by the EGM.

weights of 6 criteria are calculated with fuzzy analytical hierarchy process; thus, the upper-level factors (Kansei images)are ranked based on the weight value.

In Table 3, Kansei words (KW) or adjectives are used to represent Kansei images; KW1, KW2, KW3, KW4, KW5, and KW6, respectively, represent Easy to Recognize, Concise and displaying-visual, Striking, Distinct difference between the primary and secondary part, Futuramic, and Artless. E1 to E12 represents 12 specialists. 12 specialists are invited to conduct the paired comparison for the 6 evaluation criteria and give the evaluation value (the nine-point evaluation scale of Saaty is adopted in this questionnaire, see Table 1). Say there are $n$ criteria, a pair comparison of $n(n-1) / 2$ is required; thereby, 15 pairs are formed in paired comparison between the 6 evaluation criteria. The values in paired comparison are, respectively, $1 / 9,1 / 8, \ldots, 1 / 2,1,2, \ldots, 8,9$. The values of all specialists in the questionnaire are converted into triangular fuzzy numbers according to Table 1 , and the results are shown in Table 3.

Taking a pair of evaluation criteria KW1-KW5, for example, formula (1) can be used to calculate the agreement degree $\left(S\left(\widetilde{R}_{p}, \widetilde{R}_{q}\right)\right)$ of any two specialists. According to the agreement degree of the fuzzy evaluation values of the $p$ th and $q$ th specialists, the agreement matrix (AM) of 12 specialists for criteria KW1-KW5 can be established with formula (2) (Table 4). According to AM, the average agreement degree $A\left(E_{p}\right)$ of each specialist $p$ is calculated with formula (3); the weight of an specialist on the evaluation value of all specialists is calculated with the average agreement degree according to formula (4), that is, the relative agreement degree (RAD). The results are shown in Table 4.

Each specialist in this study has the same importance, $\mathrm{CDC}_{p}=\mathrm{RAD}_{p}$, the fuzzy numbers of all specialists for comparison between KW1-KW5 are integrated with formula (6), as shown in Table 5. Li, Mi, and Ui denote the left, middle, and right of fuzzy numbers, respectively.

Repeat the above steps and finally get the integrated fuzzy numbers of all specialists for important relationship comparison between 15 pairs of criteria. A paired comparison matrix is built for the 15 integrated fuzzy numbers with formula (7), which is shown as follows.

The 15 integrated fuzzy numbers are input into the upper triangular part of the fuzzy paired comparison matrix, its main diagonal line is the comparison of the criterion itself, and the value is 1 ; the pair comparison will have the reciprocal characteristic, the matrix consisting of the median of the fuzzy number is taken as an example (Table 6), and the ratio of the criteria KW1-KW5 is 4.3008, while the reciprocal of the ratio is 0.2325 . The lower triangular part of the paired comparison matrix is the reciprocal of the upper triangle, as shown in Table 6.

After the paired comparison matrix is established, the characteristic vector is calculated, the weight of each criterion is calculated with formulas (8)-(11) is based to calculate the eigenvalue of fuzzy-paired matrix $\widetilde{\lambda}$ and its maximum eigenvalue $\tilde{\lambda}_{\text {max }}$, as shown in Table 6 .

Saaty defined the consistency index (CI) to confirm the reasonableness of the comparison matrix of the respondents' response; Saaty suggests that it should be an acceptable error while C.I. $\leq 0.1$, and the maximum allowable range is $\mathrm{CI} \leq 0.2$. The consistency ratio (CR) refers to the ratio between the CI and RI value under the same matrix order, and the matrix evaluation value has consistency while $\mathrm{CR} \leq 0.1$. In this study, the maximum fuzzy eigenvalue $\lambda_{\max }=6.4259$ is obtained by formula (11), CI $=0.0852$ by formula (13), and $\mathrm{CR}=0.0687$ by formula (14). The results are all within the reasonable range. 
TABLE 3: 15 pirs formed in paired comparison between the 6 evaluation criteria.

\begin{tabular}{|c|c|c|c|c|c|c|c|c|c|c|c|c|}
\hline Criteria & E1 & E2 & E3 & $\mathrm{E} 4$ & E5 & $\ldots \ldots$ & E8 & E9 & E10 & E11 & E12 & Criteria \\
\hline KW1 & $\tilde{2}^{-1}$ & $\tilde{5}^{-1}$ & $\widetilde{1}$ & $\tilde{4}^{-1}$ & $\tilde{5}^{-1}$ & $\ldots \ldots \ldots$ & $\tilde{3}^{-1}$ & $\widetilde{7}$ & $\widetilde{5}^{-1}$ & $\tilde{4}$ & $\widetilde{5}$ & KW2 \\
\hline KW1 & $\widetilde{7}$ & $\widetilde{5}$ & $\tilde{6}$ & $\widetilde{3}$ & $\tilde{3}^{-1}$ & $\ldots \ldots$ & $\tilde{2}$ & $\tilde{4}$ & $\tilde{6}$ & $\tilde{6}$ & $\widetilde{7}$ & KW3 \\
\hline KW1 & $\tilde{5}^{-1}$ & $\widetilde{5}$ & $\tilde{9}$ & $\tilde{2}$ & $\tilde{6}^{-1}$ & $\ldots \ldots$ & $\tilde{3}$ & $\tilde{1}$ & $\tilde{5}^{-1}$ & $\widetilde{5}$ & $\tilde{7}$ & KW4 \\
\hline KW1 & $\tilde{4}^{-1}$ & $\widetilde{7}$ & $\widetilde{5}$ & $\widetilde{7}$ & $\widetilde{3}$ & $\ldots \ldots$ & $\widetilde{5}$ & $\tilde{6}$ & $\tilde{4}^{-1}$ & $\widetilde{5}$ & $\tilde{3}$ & KW5 \\
\hline KW1 & $\widetilde{5}$ & $\tilde{7}$ & $\widetilde{3}$ & $\tilde{7}$ & $\tilde{2}^{-1}$ & $\ldots \ldots$ & $\widetilde{8}$ & $\widetilde{3}$ & $\widetilde{5}$ & $\widetilde{5}$ & $\tilde{6}$ & KW6 \\
\hline KW2 & $\tilde{1}$ & $\tilde{4}^{-1}$ & $\tilde{1}$ & $\widetilde{5}$ & $\tilde{2}$ & $\ldots \ldots$ & $\tilde{2}$ & $\tilde{1}$ & $\tilde{4}$ & $\tilde{1}$ & $\tilde{6}^{-1}$ & KW3 \\
\hline KW2 & $\tilde{5}$ & $\tilde{5}^{-1}$ & $\tilde{3}^{-1}$ & $\tilde{3}$ & $\tilde{3}^{-1}$ & $\ldots \ldots$ & $\tilde{3}$ & $\tilde{5}^{-1}$ & $\tilde{2}$ & $\tilde{1}$ & $\widetilde{7}^{-1}$ & KW4 \\
\hline KW2 & $\widetilde{4}$ & $\tilde{1}$ & $\widetilde{3}^{-1}$ & $\widetilde{8}$ & $\widetilde{5}$ & $\ldots \ldots$ & $\tilde{4}$ & $\tilde{3}^{-1}$ & $\widetilde{3}$ & $\tilde{4}$ & $\tilde{6}$ & KW5 \\
\hline KW2 & $\tilde{4}^{-1}$ & $\tilde{3}^{-1}$ & $\tilde{1}$ & $\widetilde{8}$ & $\tilde{3}$ & $\ldots \ldots$ & $\tilde{5}$ & $\tilde{1}$ & $\widetilde{5}$ & $\tilde{5}$ & $\tilde{2}^{-1}$ & KW6 \\
\hline KW3 & $\widetilde{5}$ & $\tilde{4}$ & $\widetilde{5}$ & $\widetilde{4}^{-1}$ & $\tilde{3}^{-1}$ & $\ldots \ldots$ & $\tilde{4}^{-1}$ & $\tilde{4}^{-1}$ & $\tilde{2}$ & $\tilde{3}^{-1}$ & $\tilde{3}$ & KW4 \\
\hline KW3 & $\tilde{3}^{-1}$ & $\widetilde{5}$ & $\widetilde{1}$ & $\tilde{2}$ & $\widetilde{5}$ & $\ldots \ldots$ & $\tilde{2}$ & $\tilde{3}^{-1}$ & $\widetilde{3}$ & $\widetilde{4}$ & $\widetilde{5}^{-1}$ & KW5 \\
\hline KW3 & $\tilde{1}$ & $\tilde{4}^{-1}$ & $\widetilde{5}$ & $\tilde{4}$ & $\tilde{2}$ & $\ldots \ldots$ & $\tilde{4}$ & $\tilde{3}^{-1}$ & $\widetilde{5}$ & $\tilde{4}$ & $\tilde{3}$ & KW6 \\
\hline KW4 & $\tilde{5}^{-1}$ & $\tilde{4}^{-1}$ & $\tilde{2}^{-1}$ & $\tilde{\sigma}$ & $\widetilde{8}$ & $\ldots \ldots$ & $\tilde{3}$ & $\widetilde{6}$ & $\tilde{4}$ & $\tilde{3}$ & $\widetilde{5}$ & KW5 \\
\hline KW4 & $\tilde{4}^{-1}$ & $\tilde{2}^{-1}$ & $\widetilde{1}$ & $\widetilde{5}$ & $\tilde{4}$ & $\ldots \ldots$ & $\widetilde{5}$ & $\widetilde{6}$ & $\tilde{6}$ & $\widetilde{6}$ & $\tilde{6}^{-1}$ & KW6 \\
\hline KW5 & $\widetilde{5}^{-1}$ & $\tilde{1}$ & $\widetilde{5}$ & $\tilde{7}$ & $\tilde{3}^{-1}$ & $\ldots \ldots$ & $\tilde{2}$ & $\tilde{3}$ & $\tilde{1}$ & $\widetilde{5}$ & $\widetilde{7}^{-1}$ & KW6 \\
\hline
\end{tabular}

TABLe 4: Agreement matrix of important relationship comparison between KW1 and KW5.

\begin{tabular}{ccccccccccccc}
\hline & E1 & E2 & E3 & E4 & E5 & $\ldots$. & E9 & E10 & E11 & E12 & $A\left(E_{k}\right)$ & RAD \\
\hline E1 & 1 & 0 & 0 & 0 & 0 & $\ldots$. & 0 & 0 & 1 & 0 & 0.1270 & 0.0625 \\
E2 & 0 & 1 & 0 & 1 & 0 & $\ldots$. & 0.1429 & 0 & 0 & 0 & 0.2699 & 0.1328 \\
$\vdots$ & $\vdots$ & $\vdots$ & $\vdots$ & $\vdots$ & $\vdots$ & $\vdots$ & $\vdots$ & $\vdots$ & $\vdots$ & $\vdots$ & $\vdots$ & $\vdots$ \\
E11 & 0 & 0 & 1 & 0 & 0 & $\ldots$. & 0.1429 & 0 & 1 & 0 & 0.2699 & 0.1328 \\
E12 & 0 & 0 & 0 & 0 & 1 & $\ldots$. & 0 & 0 & 0 & 1 & 0.1429 & 0.0703 \\
\hline
\end{tabular}

TABLE 5: Integrated fuzzy numbers for comparison between KW1 and KW5.

\begin{tabular}{cccccccc}
\hline & RAD $\left(W_{i}\right)$ & $L_{i}$ & $W_{i} * L_{i}$ & $M_{i}$ & $W_{i} * M_{i}$ & $U_{i}$ & $W_{i} * U_{i}$ \\
\hline E1 & 0.0625 & $\widetilde{5}^{-1}$ & 0.0109 & $4^{-1}$ & 0.0137 & $\widetilde{3}^{-1}$ & 0.0182 \\
E2 & 0.1328 & 6 & 0.3750 & 7 & 0.4375 & 0.5000 \\
$\vdots$ & $\vdots$ & $\vdots$ & $\vdots$ & $\vdots$ & $\vdots$ & $\vdots$ \\
E11 & 0.1328 & 4 & 0.5312 & 5 & 0.6641 & 0 \\
E12 & 0.0703 & 2 & 0.1406 & 3 & 0.2109 & 0.7969 \\
& & & 3.4047 & & 4.3008 & 5 & 0.2812 \\
\hline
\end{tabular}

TABLE 6: Matrix formed by the median of integrated fuzzy numbers.

\begin{tabular}{|c|c|c|c|c|c|c|c|c|}
\hline Criteria & KW1 & KW2 & KW3 & KW4 & KW5 & KW6 & Weight & $\lambda$ \\
\hline KW1 & 1 & 1.3962 & 5.4694 & 1.4758 & 4.3008 & 5.0000 & 0.2797 & 6.7159 \\
\hline KW2 & 0.7162 & 1 & 1.3364 & 1.1338 & 3.8299 & 4.5797 & 0.1563 & 6.0518 \\
\hline KW3 & 0.1828 & 0.7483 & 1 & 1.3111 & 2.7473 & 4.0935 & 0.1383 & 6.5384 \\
\hline KW4 & 0.6776 & 0.8820 & 0.7627 & 1 & 4.8578 & 5.6269 & 0.1440 & 6.2844 \\
\hline KW5 & 0.2325 & 0.2611 & 0.3640 & 0.2059 & 1 & 3.8207 & 0.1262 & 6.5110 \\
\hline KW6 & 0.2000 & 0.2184 & 0.2443 & 0.1777 & 0.2617 & 1 & 0.0763 & 6.4539 \\
\hline \multicolumn{3}{|c|}{$\lambda_{\max }=6.4259$} & $\mathrm{CI}=0.0852$ & \multicolumn{2}{|c|}{$\mathrm{CR}=0.0687$} & & & \\
\hline
\end{tabular}

Repeat the above steps and establish a matrix composed of the left value of the integrated fuzzy number and a matrix composed of the right value. Then, the left and the right of the fuzzy weight values of the 6 criteria can be calculated, respectively, as shown in Table 7 . The left, middle, and right of the weight fuzzy value of KW1 is $0.2860,0.3299$, and 0.3643 , respectively. Then, defuzzification is performed for the weight fuzzy value of 6 criteria by formula (14), and the result is shown in Table 7 . The defuzzification weight value is ranked from top to bottom as follows: Easy to Recognize, Concise and displaying-visual, Distinct difference between the primary and secondary part, Striking, Futuramic, and Artless.

4.3. Evaluating Relationship between Key Kansei Images and Characteristics of Instrument Interface. In order to study the relationship between key Kansei images and characteristics of instrument interface, a hierarchical structure is constructed between the key Kansei images ranking first (Easy to Recognize) and the corresponding middle-level factors and 
TABLE 7: Weight and ranking of criteria.

\begin{tabular}{|c|c|c|c|c|c|c|}
\hline \multirow{2}{*}{$\begin{array}{l}\text { Criteria } \\
\text { KW1 }\end{array}$} & \multirow{2}{*}{$\begin{array}{c}\text { Upper-level item } \\
\text { Easy to Recognize }\end{array}$} & \multicolumn{3}{|c|}{$\begin{array}{l}\text { Weight fuzzy value of the } \\
\text { criteria }\end{array}$} & \multirow{2}{*}{$\begin{array}{c}\text { Defuzzification } \\
0.3268\end{array}$} & \multirow{2}{*}{$\frac{\text { Ranking }}{1}$} \\
\hline & & 0.2860 & 0.3299 & 0.3643 & & \\
\hline KW2 & Concise and displaying-visual & 0.1857 & 0.2159 & 0.2395 & 0.2137 & 2 \\
\hline KW3 & Striking & 0.1601 & 0.1485 & 0.1377 & 0.1488 & 4 \\
\hline KW4 & $\begin{array}{c}\text { Distinct difference between the primary and } \\
\text { secondary part }\end{array}$ & 0.2380 & 0.2012 & 0.1725 & 0.2039 & 3 \\
\hline KW5 & Futuramic & 0.0819 & 0.0673 & 0.0566 & 0.0686 & 5 \\
\hline KW6 & Artless & 0.0482 & 0.0372 & 0.0294 & 0.0383 & 6 \\
\hline
\end{tabular}

lower-level factors (specific characteristic), as shown in Figure 10.

The fuzzy analytic hierarchy process expert questionnaire (see, Section 3.3) is made to obtain the evaluation value of experts. The opinions of 12 experts are integrated with the SAM, and then the FAHP is based to calculate the weight of each evaluation criterion and its subcriterion (see Section 3.3). In this study, the weight value of the evaluation criterion (middle-level item) and its subcriterion (lower-level item) represents the influence degree on the optimized objective of the electric vehicle's instrument interface (the upper-level key factors). As shown in Table 8, C4 (Region segmentation of interface) is the most important in the comparison of criteria at the first hierarchy, and its weight is 0.3930; the second one is C1 (Speed information display), and its weight is 0.2378; the third one is C3 (Battery level display), and its weight is 0.1732 ; the fourth one is C2 (Font of speed information), and its weight is 0.1206; and the fifth one is C5 (Interface information), and its weight is 0.0754 . The top 3 are the weight values of C4 (Region segmentation of interface), C1 (Speed information display), and C3 (Battery level display), which causes the testees to feel stronger "Easy to Recognize" psychologically.

In the comparison of subcriteria of C4 (Region segmentation of interface), C41 (Battery gauge and speedometer are highlighted on the left and right of the interface) is the most important, and its weight is 0.5075 ; the second one is C42 (Speedometer is configured centrally and surrounded by other parameters), and its weight is 0.3818 ; and the third one is C43 (Three regions of the battery gauge, speedometer, and other functional zone are evenly distributed on the interface comparatively), and its weight is 0.1107 .

In the comparison of subcriteria of C1 (Speed information display), C13 (Simultaneously display the speed information via the scale + number) is the most important, and its weight is 0.3768; the second one is C12 (Display the speed information via figures), and its weight is 0.2888 ; the third one is C11 (Visually display the speed information via the shaded scale bars), and its weight is 0.2640; and the fourth one is $\mathrm{C} 14$ (Warn the high speed region via color blocks), and its weight is 0.0705 .

In the comparison of subcriteria of C3 (Battery level display), C31 (Battery level + percentage display) is the most important, and its weight is 0.6911 ; the second one is C33 (Indication of battery level via 10 bars), and its weight is 0.2128 ; and the third one is C32 (3D graphs of battery), and its weight is 0.0961 .

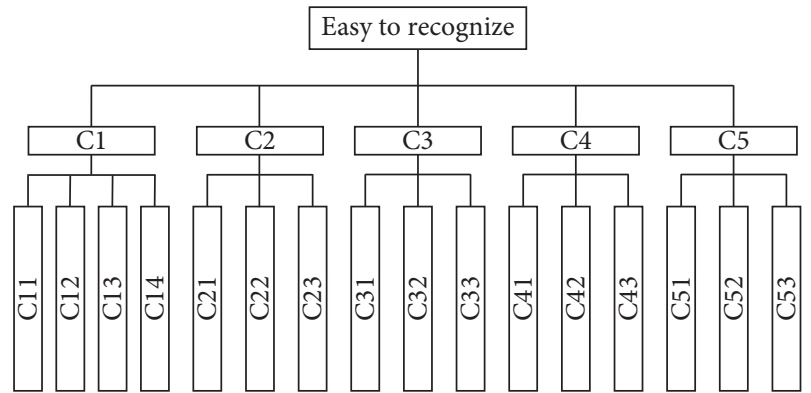

FIGURE 10: Hierarchical structure of key upper-level factors (Kansei images).

Figure 11 shows the ranking of the priority weight value of 16 subcriteria. Criterion weight $\times$ subcriterion weight $=$ priority weight. For example, the weight of criterion $\mathrm{C} 4$ is 0.3930 , the weight of subcriterion C41 is 0.5075 , then its priority weight is 0.1995 , and it ranks first in the priority weight of 16 subcriteria. Therefore, the influence degree on the psychological feelings of the testee (upper-level key factors) can be ranked, and the top 7 includes C41, C42, C31, C13, C12, C11, C21, C23, C43, and C51.

\section{Discussion}

In order to explore the availability of the instrument interface of electric vehicles and the factors related to the attraction of the interface, this study firstly derives the preference spectrum of the instrument interface from the EGM. In the second stage, it accurately calculates the weight of each upper-level attraction factor through the integration of the SAM and FAHP. The study has found that the key upper-level factor (Kansei image) of the electric vehicle instrument interface that attracts subjects is "Easily identifiable information." How to accurately identify the content in the shortest time within the limits of people's visual ability is related to two problems: visibility and readability. It enables the user to easily recognize the information on the instrument interface while driving.

In the third stage, a hierarchical association is established for the corresponding specific factors in the middle and lower level of "Easily identifiable information," and finally to calculate the weights of the 5 criteria and 16 subcriteria. The study has found that the key Kansei image "Easily identifiable information" of the user-friendly instrument interface mainly are the most important C4 (Region segmentation of 
TABLE 8: Weight of criteria and subcriteria.

\begin{tabular}{|c|c|c|c|c|c|c|c|c|c|}
\hline \multirow{5}{*}{$\begin{array}{l}\text { Criteria } \\
\mathrm{C} 1\end{array}$} & \multicolumn{3}{|c|}{ Weight between criteria (\%) } & \multirow{5}{*}{$\begin{array}{c}\text { Defuzzification } \\
0.2378\end{array}$} & \multirow{5}{*}{$\begin{array}{c}\text { Sub-criteria } \\
\text { C11 } \\
\text { C12 } \\
\text { C13 } \\
\text { C14 } \\
\end{array}$} & \multicolumn{3}{|c|}{ Weight within criteria (\%) } & \multirow{2}{*}{$\begin{array}{c}\text { Defuzzification } \\
0.2640\end{array}$} \\
\hline & & & & & & 0.2214 & 0.2635 & 0.3070 & \\
\hline & & & & & & 0.2618 & 0.2910 & 0.3134 & 0.2888 \\
\hline & 0.1839 & 0.2360 & 0.2935 & & & 0.4333 & 0.3761 & 0.3209 & 0.3768 \\
\hline & & & & & & 0.0835 & 0.0694 & 0.0586 & 0.0705 \\
\hline \multirow{3}{*}{$\mathrm{C} 2$} & & & & & $\mathrm{C} 21$ & 0.3872 & 0.4854 & 0.5673 & 0.4800 \\
\hline & 0.1083 & 0.1203 & 0.1332 & 0.1206 & $\mathrm{C} 22$ & 0.1117 & 0.1090 & 0.1092 & 0.1100 \\
\hline & & & & & $\mathrm{C} 23$ & 0.5011 & 0.4056 & 0.3235 & 0.4101 \\
\hline \multirow{3}{*}{ C3 } & & & & & $\mathrm{C} 31$ & 0.6338 & 0.6973 & 0.7423 & 0.6911 \\
\hline & 0.1710 & 0.1734 & 0.1752 & 0.1732 & C32 & 0.0957 & 0.0958 & 0.0968 & 0.0961 \\
\hline & & & & & $\mathrm{C} 33$ & 0.2705 & 0.2069 & 0.1609 & 0.2128 \\
\hline \multirow{3}{*}{$\mathrm{C} 4$} & & & & & $\mathrm{C} 41$ & 0.4600 & 0.5107 & 0.5518 & 0.5075 \\
\hline & 0.4476 & 0.3962 & 0.3352 & 0.3930 & $\mathrm{C} 42$ & 0.4047 & 0.3816 & 0.3591 & 0.3818 \\
\hline & & & & & $\mathrm{C} 43$ & 0.1353 & 0.1078 & 0.0891 & 0.1107 \\
\hline \multirow{3}{*}{ C5 } & & & & & C51 & 0.5311 & 0.5792 & 0.6155 & 0.5752 \\
\hline & 0.0891 & 0.0741 & 0.0629 & 0.0754 & C52 & 0.2942 & 0.2898 & 0.2800 & 0.2880 \\
\hline & & & & & C53 & 0.1747 & 0.1311 & 0.1045 & 0.1367 \\
\hline
\end{tabular}

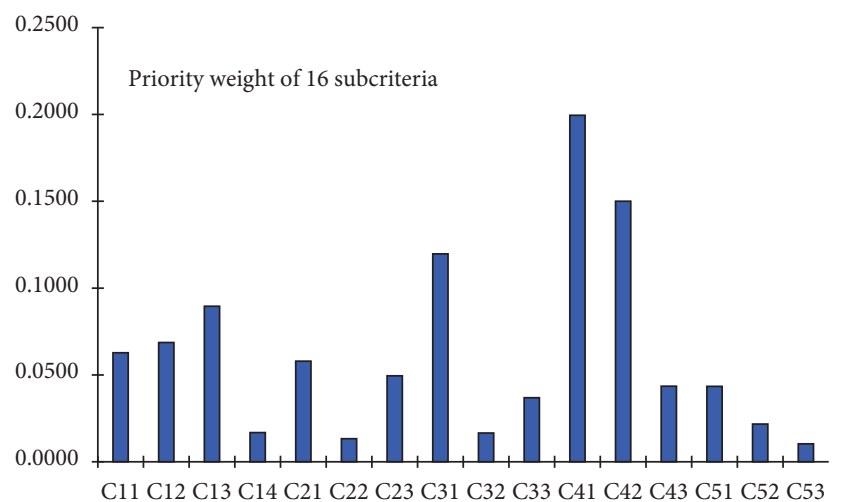

FIgURE 11: Ranking in priority weight of subcriteria.

interface), followed by $\mathrm{C} 1$ (Speed information display) and C3 (Battery level display). In the 16 subcriteria, the top 7 of specific factors that allows the subjects to produce "Easily identifiable information" to the instrument interface are C41, C42, C31, C13, C12, C11, and C21.

The importance of C4 (Region segmentation of interface) is ranked first. In the comparison of subcriteria of C4, C41 (Battery gauge and speedometer are highlighted on the left and right of the interface) is the most important and ranks first in the priority of the 16 subcriteria. (1) Currently, the electric vehicle instrument is developing towards the direction of the integrated information system, the instrument interface can add some other information, such as GPS, Bluetooth, light headlights, and network signals, in addition to the main information highlighted, such as speed, power, mileage, and direction indicators. (2) The interface space of the instrument is limited. It is a big challenge to make the information of the interface easy to identify in such a small range. This requires the interface planning of the instrument first highlight the information of the speedometer and the electricity meter and avoid the secondary information of the interface to interfere with the identification of the main information; otherwise, the driver cannot quickly and accurately identify important instrument information. Based on the highlighting of important information, consider the matching of secondary information in the planning of the instrument interface. The instrument interface planning, which first highlights the speedometer and the fuel gauge, does not distract the subjects and is more likely to be recognized by people than the complex interface.

The importance of C3 (Battery level display) ranks the third. In the comparison of its subcriteria, C31 (Battery level + percentage display) is the most important and C31 ranks the third among the priority weights of the 16 subcriteria. For electric vehicles, the effective estimation of the remaining battery display and its display method can avoid the trouble of calling rescue on the road. Use 10 battery level + percentage to show that the battery power can convey the information status of the battery more accurately. The inspiration of the study results to the optimization design of the instrument interface are the electricity meter uses the design pattern of the battery graphic symbol and the internal uses the 10 battery level + percentage display to show that the battery power can make the display of the electricity accurately and quickly recognized.

The importance of C1 (Speed information display) ranks the second. In the comparison of its subcriteria, C13 (Simultaneously display the speed information via the scale + number) is the most important, followed by $\mathrm{C} 12$ (Display the speed information via figures). The priority weights of $\mathrm{C} 13$ and $\mathrm{C} 12$ rank the 4th and 5th among the 16 subcriteria. (1) The speed information is conveyed by digital expression, which makes it simpler, faster, and more accurate for the subjects to read speed information. For pointer type instruments, the subjects need to see and locate the scale position pointed by the pointer first and then look at the number pointed, which makes the process of identifying information cumbersome compared with the digital speedometer. (2) A number of speedometers generally use large fonts so that it can be easily identified by users, which form a strong contrast between the speedometer and other elements, making the speedometer more striking. (3). 
Scale + digital display speed information allows the driver use the scale to get an overview of whether the speed is in a safe range or accurately identify the speed of the vehicle.

\section{Conclusion}

In this study, optimization design of the instrument interface of electric vehicles based on Kansei image preferences is taken as a case. By making experimental samples, the author invites the subjects to conduct simulation experiments. After the experiments, the subjects are invited to rate Kansei image preferences of the experimental samples. According to statistical analysis, six Kansei images are obtained: "Easily identifiable information," "Information content with clear priorities," "Concise and intuitive display," "Striking," "New interface design," and "Simple." In the second stage, this study revised the evaluation results of EMG qualitative analysis. By integrating the SAM and FAHP, the weights of each upper-level factor were calculated. The first three Kansei images "Easily identifiable information," "interface of instrument with concise and intuitive display," and "Information content with clear priorities" were sorted and "Easily identifiable information" was the preferred factor for the optimization design of the instrument interface. In the third stage, by integrating the SAM and FAHP, the important weights of the 5 criteria and 16 subcriteria to the interface optimization target were calculated, and the important specific characteristics of usability and interface attractiveness were obtained. The study results can assist designers of the electric vehicle instrument to optimize the interface design. The conclusions of this paper mainly are as follows:

(1) The process of extracting the user's psychological feeling through the EGM is to obtain the upper, middle, and lower factors by statistical sorting according to the number of times mentioned by the subjects, so it is a qualitative analysis process to determine the factors that attract the product through the EGM. In this study, the attractive factors obtained by the EGM are taken as a basic evaluation and the matrix is further constructed by FAHP, and the weight relationship between the upper, middle, and lower factors is obtained, which is more effective than previous studies extracting users' Kansei only based on the EGM of the Miryoku Engineering.

(2) The author uses the upper, middle, and lower factors obtained through the EGM as the evaluation criteria and subcriteria. First, experts are invited to make a pairwise comparison between each criterion and subcriteria. Then, the SAM is used to integrate the opinions of each expert, and then the FAHP is used to calculate the weights of each criterion and subcriteria and prioritize. Compared with the traditional weighting methods AHP and FAHP, this study helps vehicle designers and engineers to more accurately analyze users' Kansei factors.

(3) The paper has studied the relationship between key upper-level factors (Kansei images) and specific features of the instrument interface. This study has established a hierarchical structure by ranking the first key upper factor and its corresponding middle factors and lower factors (specific features) and obtained important specific factors that affect the optimization of the instrument interface. The study provides an important basis for vehicle designers and engineers to provide information on how to optimize the instrument interface format and design of reconfigurable displays.

Because of some limitations, the study in this paper needs to continue to improve. First of all, in the process of the EGM, each subject has different understandings of the descriptions in the questionnaires; therefore, errors are inevitable. The researchers need to correctly analyze the subjects' language and meaning. However, the language described by the subjects cannot express the semantic space of the meter in detail, so there may be errors in determining the factors of the subjects' Kansei. Secondly, in the preference test of the electric vehicle instrument sample, some sample brands have higher visibility. Even if the logo is removed, some consumers may have subjective tendencies towards the brand during testing. Thirdly, the study results are not fixed but vary with Kansei factors such as the development of the vehicles, time, and different ethnic groups.

\section{Data Availability}

The data used to support the findings of this study are included within the article.

\section{Conflicts of Interest}

The authors declare that there are no conflicts of interest regarding the publication of this paper.

\section{Acknowledgments}

The authors appreciate the experts and designers who offer great help.

\section{References}

[1] M. Ma, Y. Chen, and S. Li, "How to build design strategy for attractiveness of new products (DSANP)," Advances in Information Sciences and Service Sciences, vol. 3, no. 11, pp. 17-26, 2011.

[2] C. Alppay and N. Bayazit, "An ergonomics based design research method for the arrangement of helicopter flight instrument panels," Applied Ergonomics, vol. 51, pp. 85-101, 2015.

[3] F. Bellotti, A. De Gloria, A. Poggi, L. Andreone, S. Damiani, and P. Knoll, "Designing configurable automotive dashboards on liquid crystal displays," Cognition, Technology \& Work, vol. 6, no. 4, pp. 247-265, 2004.

[4] C. Owsley, G. McGwin Jr., and T. Seder, "Older drivers' attitudes about instrument cluster designs in vehicles," Accident Analysis \& Prevention, vol. 43, no. 6, pp. 2024-2029, 2011.

[5] Z. Gibson, J. Butterfield, and A. Marzano, "User-centered design criteria in next generation vehicle consoles," Procedia CIRP, vol. 55, pp. 260-265, 2016. 
[6] D. Gkouskos, Joyride! towards User Experience Design for InVehicle Systems, Chalmers University of Technology, Gothenburg, Sweden, 2014.

[7] C. Xue, J. Li, H. Wang, and Y. Niu, "Effects of target and distractor saturations on the cognitive performance of an integrated display interface," Chinese Journal of Mechanical Engineering, vol. 28, no. 1, pp. 208-216, 2015.

[8] J. G. Ells and R. E. Dewar, "Rapid comprehension of verbal and symbolic traffic sign messages," Human Factors: The Journal of the Human Factors and Ergonomics Society, vol. 21, no. 2, pp. 161-168, 1979.

[9] M. Borrus and J. A. Hart, "Display's the thing: the real stakes in the conflict over high-resolution displays," Journal of Policy Analysis and Management, vol. 13, no. 1, pp. 21-54, 1994.

[10] S.-F. Liu, A.-S. Lee, Y.-W. Liang, and J.-Y. Lin, "A study on the influence of the designs and configurations of the displays on a motorcycle instrument panel on cognitive effects in Taiwan," Journal of Information and Optimization Sciences, vol. 33, no. 6, pp. 685-700, 2012.

[11] M. Nagamachi, "Kansei engineering: a new ergonomic consumer-oriented technology for product development," International Journal of Industrial Ergonomics, vol. 15, no. 1, pp. 3-11, 1995.

[12] K. H. Chen, K. S. Shen, and M. Y. Ma, "The functional and usable appeal of facebook SNS games," Internet Research, vol. 22, no. 4, pp. 467-481, 2012.

[13] J. J. Buckley, "Fuzzy hierarchical analysis," Fuzzy Sets and Systems, vol. 17, no. 3, pp. 233-247, 1985.

[14] W. B. Ahmed and B. Yannou, "A bayesian learning of probabilistic relations between perceptual attributes and technical characteristics of car dashboards to construct a perceptual evaluation model," International Journal of Product Development, vol. 7, no. 1/2, pp. 47-72, 2009.

[15] T. Jindo and K. Hirasago, "Application studies to car interior of Kansei engineering," International Journal of Industrial Ergonomics, vol. 19, no. 2, pp. 105-114, 1997.

[16] S. Smith and S.-H. Fu, "The relationships between automobile head-up display presentation images and drivers' Kansei," Displays, vol. 32, no. 2, pp. 58-68, 2011.

[17] C. H. Ho and K. C. Hou, "Exploring the attractive factors of app icons," KSII Transactions on Internet and Information Systems, vol. 9, pp. 2251-2270, 2015.

[18] M. Y. Ma, Y. C. Chen, and S. R. Li, "How to build design strategy for attractiveness of new products (DSANP)," Advances in Information Sciences and Service Sciences, vol. 3, pp. 17-26, 2011.

[19] K. S. Shen, "Measuring the sociocultural appeal of SNS games in Taiwan," Internet Research, vol. 23, no. 3, pp. 372-392, 2013.

[20] M. Ujigawa, G. Maruyama, and J. Sanui, "Development of evaluation grid method using electronic-mail: a study on users' preference for gas stations," Journal of Architecture and Planning (Transactions of AIJ), vol. 64, no. 518, pp. 75-80, 1999.

[21] X. Kang, M. Yang, Y. Wu et al., "Integrating evaluation grid method and fuzzy quality function deployment to new product development," Mathematical Problems in Engineering, vol. 2018, Article ID 2451470, 15 pages, 2018.

[22] J. Sanui, "Visualization of users' requirements: introduction of the evaluation grid method," in Proceedings of the 3rd Design $\&$ Decision Support Systems in Architecture \& Urban Planning Conference, pp. 365-374, Spa, Belgium, August 1996.

[23] K. S. Shen, K. H. Chen, C. C. Liang et al., "Measuring the functional and usable appeal of crossover B-car interiors,"
Human Factors and Ergonomics in Manufacturing \& Service Industries, vol. 25, pp. 106-122, 2012.

[24] J. Park, J.-H. Kim, E.-J. Park, and S. M. Ham, “Analyzing user experience design of mobile hospital applications using the evaluation grid method," Wireless Personal Communications, vol. 91, no. 4, pp. 1591-1602, 2016.

[25] T. L. Saaty, The Analytic Hierarchy Process, McGraw-Hill International, New York, NY, USA, 1980.

[26] K. Chakraborty, S. Mondal, and K. Mukherjee, "Analysis of product design characteristics for remanufacturing using fuzzy AHP and axiomatic design," Journal of Engineering Design, vol. 28, no. 5, pp. 338-368, 2017.

[27] S. M. Sapuan, J. Y. Kho, E. S. Zainudin et al., "Materials selection for natural fiber reinforced polymer composites using analytical hierarchy process," Indian Journal of Engineering and Materials Sciences, vol. 18, pp. 255-267, 2011.

[28] R. Zhou and A. H. S. Chan, "Using a fuzzy comprehensive evaluation method to determine product usability: a test case," Work, vol. 56, no. 1, pp. 21-29, 2017.

[29] R. Zhou and A. H. S. Chan, "Using a fuzzy comprehensive evaluation method to determine product usability: a proposed theoretical framework," Work, vol. 56, no. 1, pp. 9-19, 2017.

[30] P. J. M. Van Laarhoven and W. Pedrycz, "A fuzzy extension of Saaty's priority theory," Fuzzy Sets and Systems, vol. 11, no. 1-3, pp. 229-241, 1983.

[31] H.-Y. Chen, "Development of perfume bottle visual design model using fuzzy analytic hierarchy process," Art and Design Review, vol. 5, no. 1, pp. 13-25, 2017.

[32] S. E. Alptekin, "A fuzzy decision support system for digital camera selection based on user preferences," Expert Systems with Applications, vol. 39, no. 3, pp. 3037-3047, 2012.

[33] J. C. Tu and C. L. Hu, "Applying the fuzzy analytic hierarchy process to construct the product innovative service system of wedding photography apparel," Mathematical Problems in Engineering, vol. 2015, Article ID 171204, 12 pages, 2015.

[34] H. M. Hsu and C. T. Chen, "Aggregation of fuzzy opinions under group decision making," Fuzzy Sets and Systems, vol. 79 , no. 3, pp. 279-285, 1996.

[35] Y. Wu and J. Cheng, "Continuous fuzzy kano model and fuzzy AHP model for aesthetic product design: case study of an electric scooter," Mathematical Problems in Engineering, vol. 2018, Article ID 4162539, 13 pages, 2018. 


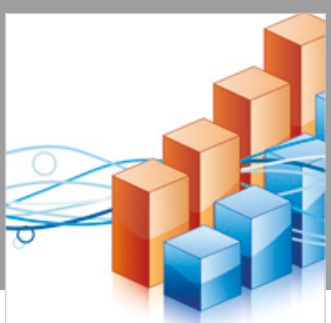

Advances in

Operations Research

\section{-n-m}
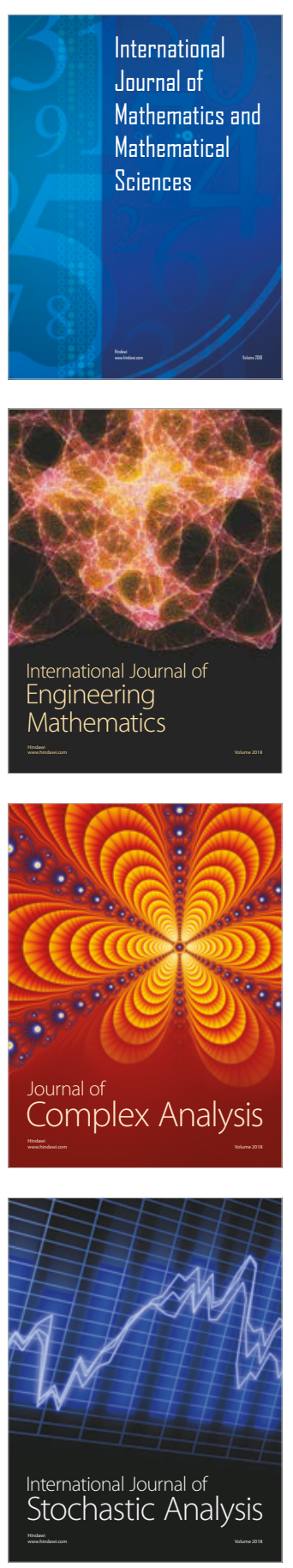
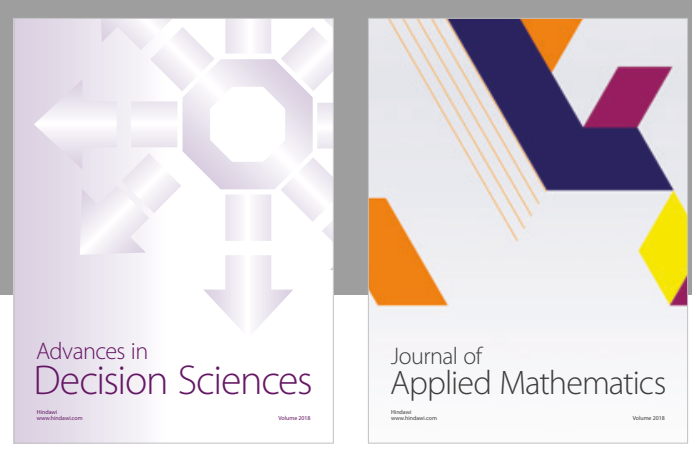

Journal of

Applied Mathematics
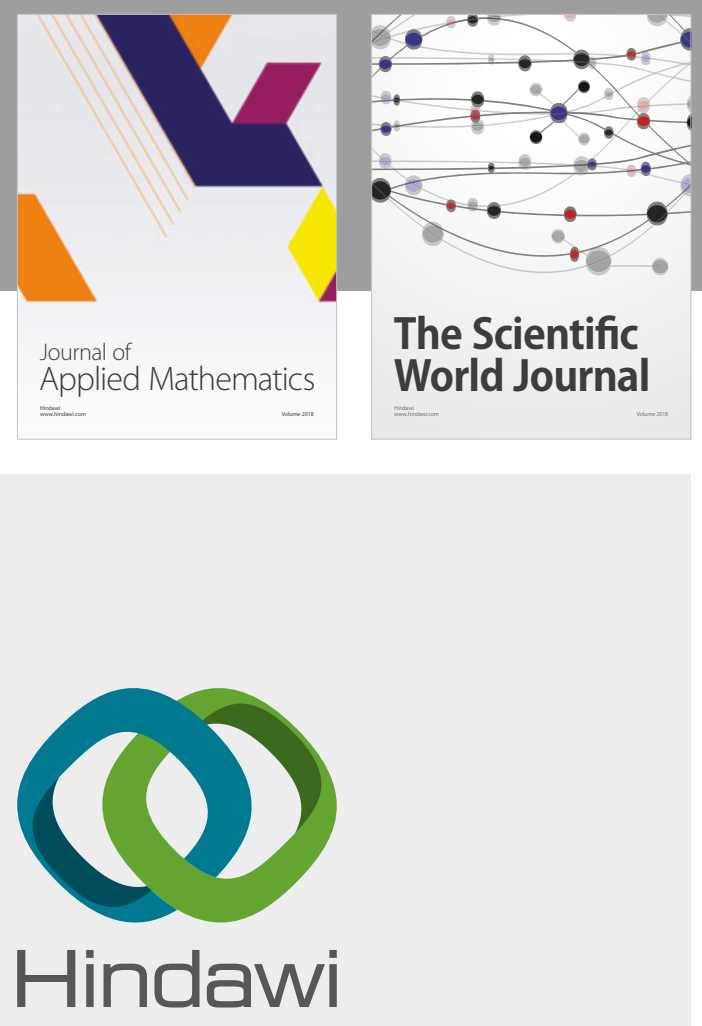

Submit your manuscripts at

www.hindawi.com

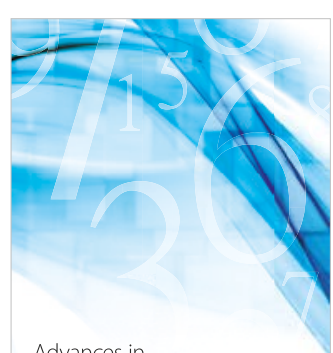

Advances in
Numerical Analysis
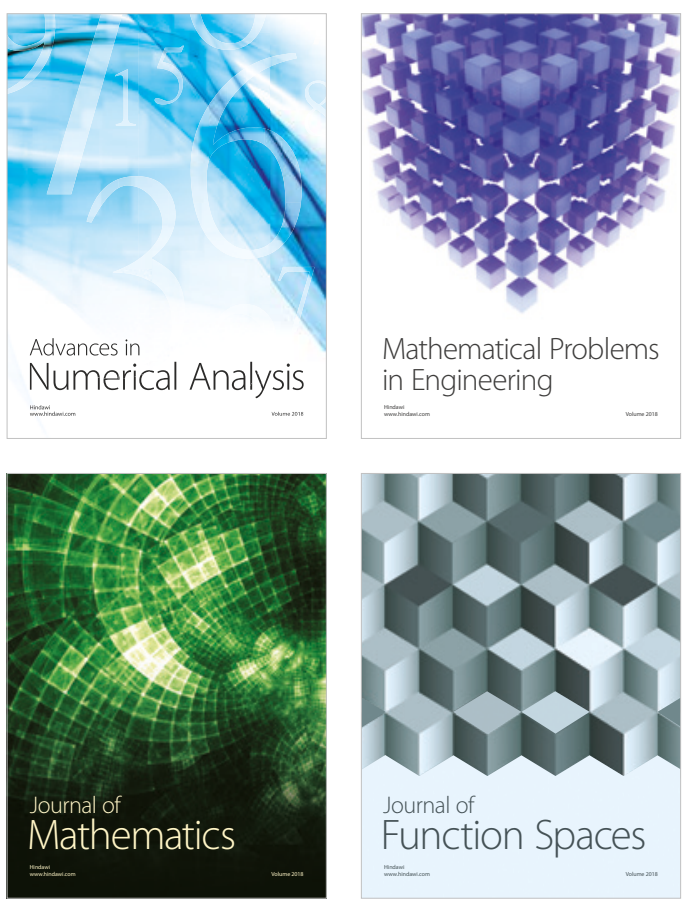

Mathematical Problems in Engineering

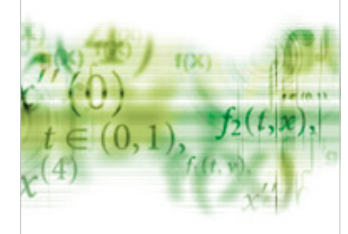

International Journal of

Differential Equations

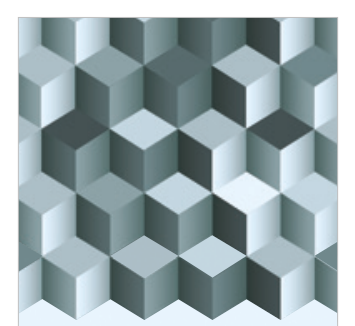

Journal of

Function Spaces

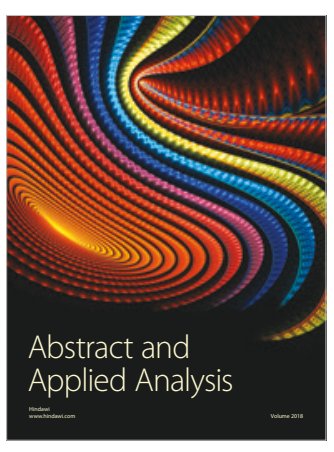

The Scientific

World Journal

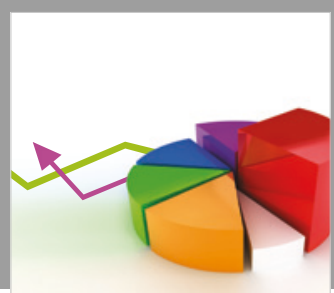

Journal of

Probability and Statistics
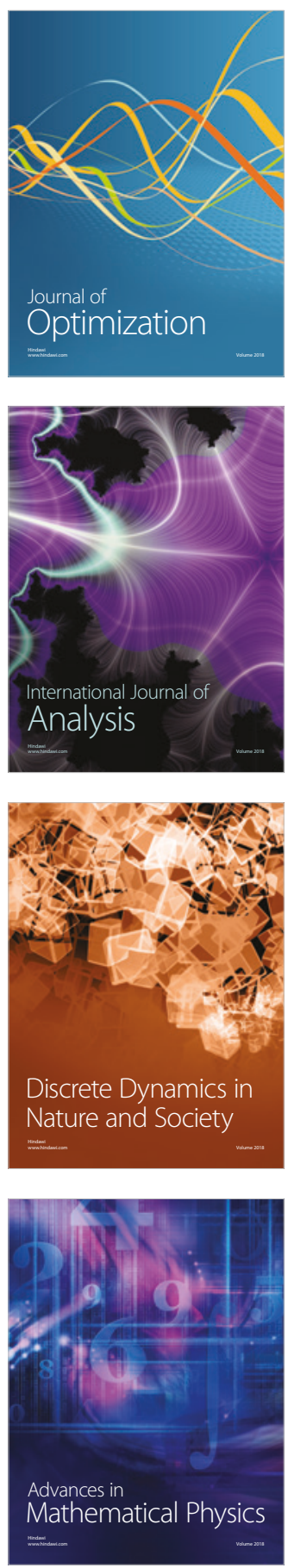